\title{
Positroids, non-crossing partitions, and positively oriented matroids
}

\author{
Federico Ardila $\left.\right|^{1 k}$ \\ Felipe Rincón" \\ Lauren Williams 非
}

\author{
${ }^{1}$ Mathematics Department, San Francisco State University, USA \\ ${ }^{2}$ Mathematics Institute, University of Warwick, United Kingdom \\ ${ }^{3}$ Mathematics Department, University of California, Berkeley, USA
}

\begin{abstract}
We investigate the role that non-crossing partitions play in the study of positroids, a class of matroids introduced by Postnikov. We prove that every positroid can be constructed uniquely by choosing a non-crossing partition on the ground set, and then freely placing the structure of a connected positroid on each of the blocks of the partition. We use this to enumerate connected positroids, and we prove that the probability that a positroid on $[n]$ is connected equals $1 / e^{2}$ asymptotically. We also prove da Silva's 1987 conjecture that any positively oriented matroid is a positroid; that is, it can be realized by a set of vectors in a real vector space. It follows from this result that the positive matroid Grassmannian (or positive MacPhersonian) is homeomorphic to a closed ball.

Résumé. Nous étudions le rôle des partitions sans croisements dans l'étude des positroïdes, une classe de matroïdes introduite par Postnikov. On montre que chaque positroïde peut être construit de manière unique par le choix d'une partition sans croisements de l'ensemble $[n]$ ainsi que le choix d'un positroide connexe pour chacun des blocs de la partition. Nous utilisons ce résultat pour énumérer les positroïdes connexes, et nous prouvons que la probabilité qu'un positroïde sur $[n]$ soit connexe est asymptotiquement égale à $1 / e^{2}$. Nous prouvons aussi une conjecture de 1987 dûe à da Silva : tout matroïde orienté positivement est un positroïde; autrement dit, il peut être réalisé par un ensemble de vecteurs dans un espace vectoriel réel. Il découle de ce résultat que la Grassmannienne matroïde positive (ou MacPhersonienne positive) est homéomorphe à un boule fermée.
\end{abstract}

Keywords: positroid, non-crossing partition, matroid polytope, oriented matroid, matroid Grassmannian.

\section{Introduction}

Matroid theory was introduced in the 1930s as a combinatorial model that keeps track of, and abstracts, the dependence relations among a set of vectors. It has become an extremely powerful model in many other contexts, but its connections to linear algebra are still the subject of very interesting research today.

\footnotetext{
*Email: federicoesfsu.edu. Partially supported by the National Science Foundation CAREER Award DMS-0956178 and the SFSU-Colombia Combinatorics Initiative

†Email: e.f.rincon@warwick.ac.uk. Supported by the EPSRC grant EP/I008071/1.

‡Email: williams@math. berkeley . edu. Partially supported by the National Science Foundation CAREER award DMS1049513.
}

1365-8050 @ 2014 Discrete Mathematics and Theoretical Computer Science (DMTCS), Nancy, France 
Not every matroid arises from linear algebra, and one of the early hopes in the area was to discover the "missing axiom" which characterizes the matroids that can be realized by a set of vectors. It is now believed that this is not a reasonable goal [MNW, Vám78], or in Vámos's words, that "the missing axiom of matroid theory is lost forever".

A positroid is a matroid on an ordered set which can be realized by the columns of a full rank $d \times n$ real matrix such that all its maximal minors are nonnegative. Such matroids were first considered by Postnikov [Posb] in his study of the totally nonnegative part of the Grassmannian. He unveiled their elegant combinatorial structure, and showed they are in bijection with several interesting classes of combinatorial objects, including Grassmann necklaces, decorated permutations, I-diagrams, and equivalence classes of plabic graphs. They have recently been found to have very interesting connections with cluster algebras $[\mathrm{Sco} 06]$ and quantum field theory $\left[\mathrm{AHBC}^{+}\right]$.

In this extended abstract we investigate the role that non-crossing partitions play in the study of positroids. In Theorem 3.2 we prove that the connected components of a positroid form a non-crossing partition of its ground set. Conversely, each positroid on $[n]$ can be uniquely constructed by choosing a non-crossing partition $\left(S_{1}, \ldots, S_{t}\right)$ of $[n]$, and then putting the structure of a connected positroid on each block $S_{i}$. The first statement was also discovered in [OPS], where it is stated without proof, and in [For].

Our structural result allows us to enumerate connected positroids, as described in Theorem 4.6 Along the way, we show in Corollary 3.8 that the connected positroids on $[n]$ are in bijection with the stabilizedinterval-free permutations on $[n]$; that is, the permutations $\pi$ such that $\pi(I) \neq I$ for all intervals $I \subsetneq[n]$. We then show in Theorem 4.7 that the proportion of positroids on $[n]$ which are connected is equal to $1 / e^{2}$ asymptotically. This result is somewhat surprising in light of the conjecture [MNWW11] that "most matroids are connected"; more specifically, that as $n$ goes to infinity, the ratio of connected matroids on $[n]$ to matroids on $[n]$ tends to 1 .

We also investigate matroid polytopes associated to positroids. A general matroid polytope for a matroid on the ground set $[n]$ can be described by using $2^{n}$ inequalities; in contrast, a positroid polytope for a rank $d$ positroid on $[n]$ can be described using $d n+n$ inequalities. In Theorem 2.5 we prove a characterization of positroid polytopes, which we learned from Alex Postnikov [Posa] and will also appear in [LP]. More strongly, we show in Theorem 3.11 that the face poset of a positroid polytope naturally embeds in a poset of weighted non-crossing partitions.

Oriented matroid theory was introduced in the 1970s as a model for real hyperplane arrangements; or equivalently, for the dependence relations among a set of real vectors together with their signs. Again, the problem of characterizing which oriented matroids actually come from real hyperplane arrangements is intractable. Even for orientations of uniform matroids, there is no finite set of excluded minors for realizability [BS89] [BLVS ${ }^{+99}$, Theorem 8.3.5].

The problem of (oriented) matroid realizability over the field $\mathbb{Q}$ of rational numbers is particularly hard. Sturmfels proved [Stu87] that the existence of an algorithm for deciding if any given (oriented) matroid is realizable over $\mathbb{Q}$ is equivalent to the existence of an algorithm for deciding the solvability of arbitrary Diophantine equations within the field of rational numbers. It is also equivalent to the existence of an algorithm that decides if a given lattice is isomorphic to the face lattice of a convex polytope in rational Euclidean space. Despite much interest, all of these problems remain open.

Positively oriented matroids were introduced by Ilda da Silva in 1987. They are oriented matroids for which all bases have a positive orientation. The motivating example is the uniform positively oriented matroid $\mathcal{C}^{n, r}$, which is realized by the vertices of the cyclic polytope $C^{n, r}$ [Bla77, LV75]. Da Silva studied the combinatorial properties of positively oriented matroids, and proposed the following conjecture. 
Conjecture 1.1 (da Silva, 1987 [dS87]) Every positively oriented matroid is realizable.

Every positroid gives rise to a positively oriented matroid, and da Silva's Conjecture 1.1 is the converse statement. This is one of our main theorems.

Theorem 5.5 Every positively oriented matroid is a positroid, and is therefore realizable over $\mathbb{Q}$.

There is a natural partial order on oriented matroids called specialization. In [Mac93], motivated by his theory of combinatorial differential manifolds, MacPherson introduced the matroid Grassmannian (also called the MacPhersonian) $\operatorname{MacP}(d, n)$, which is the poset of rank $d$ oriented matroids on $[n]$ ordered by specialization. He showed that $\operatorname{MacP}(d, n)$ plays the same role for matroid bundles as the ordinary Grassmannian plays for vector bundles, and pointed out that the geometric realization of the order complex $\|\operatorname{MacP}(d, n)\|$ of $\operatorname{MacP}(d, n)$ is homeomorphic to the real Grassmannian $\operatorname{Gr}(d, n)$ if $d$ equals $1,2, n-2$, or $n-1$. "Otherwise, the topology of the matroid Grassmannian is mostly a mystery."

Since MacPherson's work, some progress on this question has been made, most notably by Anderson [And99], who obtained results on homotopy groups of the matroid Grassmannian, and by Anderson and Davis [AD02], who constructed maps between the real Grassmannian and the matroid Grassmannian showing that philosophically, there is a splitting of the map from topology to combinatorics - and thereby gained some understanding of the mod 2 cohomology of the matroid Grassmannian. However, many open questions remain.

We define the positive matroid Grassmannian or positive MacPhersonian $\mathrm{MacP}^{+}(d, n)$ to be the poset of rank $d$ positively oriented matroids on $[n]$, ordered by specialization. By Theorem 5.5 , each positively oriented matroid can be realized by an element of the positive Grassmannian $\mathrm{Gr}^{+}(d, n)$. Combining this fact with results of the third author [Wil07], we obtain the following result.

Theorem 6.4 The positive matroid Grassmannian $\left\|\mathrm{MacP}^{+}(d, n)\right\|$ is homeomorphic to a closed ball.

\section{Positroids and matroid polytopes}

A matroid is a combinatorial object that unifies several notions of independence. Among the many equivalent ways of defining a matroid we will adopt the point of view of bases, which is one of the most convenient for the study of positroids and matroid polytopes. We refer the reader to [Ox192] for a more in-depth introduction to matroid theory.

Definition 2.1 $A$ matroid $M$ is a pair $(E, \mathcal{B})$ consisting of a finite set $E$ and a nonempty collection of subsets $\mathcal{B}=\mathcal{B}(M)$ of $E$, called the bases of $M$, which satisfy the basis exchange axiom:

- If $B_{1}, B_{2} \in \mathcal{B}$ and $b_{1} \in B_{1}-B_{2}$, then there exists $b_{2} \in B_{2}-B_{1}$ such that $\left(B_{1}-b_{1}\right) \cup b_{2} \in \mathcal{B}$.

Example 2.2 Let $A$ be a $d \times n$ matrix of rank $d$ with entries in a field $K$, and denote its columns by $\mathbf{a}_{1}, \mathbf{a}_{2}, \ldots, \mathbf{a}_{n} \in K^{d}$. The subsets $B \subseteq[n]$ for which the columns $\left\{\mathbf{a}_{i} \mid i \in B\right\}$ form a linear basis for $K^{d}$ are the bases of a matroid $M(A)$ on the set $[n]$. Matroids arising in this way are called realizable, and motivate much of the theory of matroids.

The following geometric representation of a matroid will be useful in our study of positroids.

Definition 2.3 Given a matroid $M=([n], \mathcal{B})$, the (basis) matroid polytope $\Gamma_{M}$ of $M$ is the convex hull of the indicator vectors of the bases of $M$ :

$$
\Gamma_{M}:=\operatorname{convex}\left\{e_{B} \mid B \in \mathcal{B}\right\} \subset \mathbb{R}^{n},
$$


where $e_{B}:=\sum_{i \in B} e_{i}$, and $\left\{e_{1}, \ldots, e_{n}\right\}$ is the standard basis of $\mathbb{R}^{n}$.

We now introduce a special class of realizable matroids studied by Postnikov in [Posb].

Definition 2.4 Suppose $A$ is a $d \times n$ matrix of rank $d$ with real entries such that all its maximal minors are nonnegative. Such a matrix $A$ is called totally nonnegative, and the realizable matroid $M(A)$ associated to it is called a positroid. In fact, it follows from the work of Postnikov that any positroid can be realized by a totally nonnegative matrix with entries in $\mathbb{Q}[$ [Posb. Theorem 4.12].

We will make use of the following notation. Given $k, \ell \in[n]$, we define the (cyclic) interval $[k, \ell]$ to be the set

$$
[k, \ell]:= \begin{cases}\{k, k+1, \ldots, \ell\} & \text { if } k \leq \ell, \\ \{k, k+1, \ldots, n, 1, \ldots, \ell\} & \text { if } \ell<k .\end{cases}
$$

We will often put a total order on a cyclic interval in the natural way.

The following key result gives a characterization of positroids in terms of their matroid polytopes. It will also appear in an upcoming preprint of Lam and Postnikov [LP].

Theorem 2.5 A matroid $M$ of rank $d$ on $[n]$ is a positroid if and only if its matroid polytope $\Gamma_{M}$ can be described by the equality $x_{1}+\cdots+x_{n}=d$ and inequalities of the form

$$
\sum_{\ell \in[i, j]} x_{\ell} \leq a_{i j}, \text { with } i, j \in[n] .
$$

\section{Positroids, connected positroids, and non-crossing partitions}

In this section we illustrate the role that non-crossing partitions play in the theory of positroids.

Definition 3.1 We say that two disjoint subsets $T$ and $T^{\prime}$ of $[n]$ are non-crossing if there is a cyclic interval of $[n]$ containing $T$ and disjoint from $T^{\prime}$ (and vice versa). If $S$ is a partition $[n]=S_{1} \sqcup \cdots \sqcup S_{t}$ of $[n]$ into pairwise disjoint non-empty subsets, we say that $S$ is a non-crossing partition if any two parts $S_{i}$ and $S_{j}$ are non-crossing. Let $N C_{n}$ denote the set of non-crossing partitions of $[n]$.

Theorem 3.2 Let $M$ be a positroid on $[n]$ and let $S_{1}, S_{2}, \ldots, S_{t}$ be the ground sets of the connected components of $M$. Then $\Pi_{M}=\left\{S_{1}, \ldots, S_{t}\right\}$ is a non-crossing partition of $[n]$, called the non-crossing partition of $M$. Conversely, if $S_{1}, S_{2}, \ldots, S_{t}$ form a non-crossing partition of $[n]$ and $M_{1}, M_{2}, \ldots, M_{t}$ are connected positroids on $S_{1}, S_{2}, \ldots, S_{t}$, respectively, then $M_{1} \oplus \cdots \oplus M_{t}$ is a positroid.

The first half of Theorem 3.2 was also stated without proof by Oh, Postnikov, and Speyer in [OPS], and will also appear in Ford's preprint [For].

In his study of the totally nonnegative part of the Grassmannian [ [Posb], Postnikov showed that positroids are in bijection with certain combinatorial objects called decorated permutations.

Definition 3.3 A decorated permutation of the set $[n]$ is a bijection $\pi:[n] \rightarrow[n]$ whose fixed points are colored either "clockwise" or "counterclockwise."

The following results, describing direct sums and connectivity in terms of decorated permutations, are also anticipated in [OPS]. 
Definition 3.4 Suppose $S_{1}$ and $S_{2}$ are disjoint sets. If $\pi_{1}$ is a decorated permutation of $S_{1}$ and $\pi_{2}$ is a decorated permutation of $S_{2}$, the direct sum $\pi_{1} \oplus \pi_{2}$ is the decorated permutation of the set $S_{1} \sqcup S_{2}$ such that $\left.\pi\right|_{S_{1}}=\pi_{1}$ and $\left.\pi\right|_{S_{2}}=\pi_{2}$.

Proposition 3.5 Let $M_{1}, \ldots, M_{t}$ be positroids on the ground sets $S_{1}, \ldots, S_{t}$, respectively, and suppose $\left\{S_{1}, \ldots, S_{t}\right\}$ is a non-crossing partition of $[n]$. Let $\pi_{i}$ be the decorated permutation of $S_{i}$ associated to $M_{i}$, for $i=1, \ldots, t$. Then the decorated permutation associated to the positroid $M_{1} \oplus \cdots \oplus M_{t}$ is the direct sum $\pi_{1} \oplus \cdots \oplus \pi_{t}$.

Corollary 3.6 Let $M$ be a positroid on $[n]$, and let $\pi$ be is its corresponding decorated permutation. Then the non-crossing partition $\Pi_{M}$ associated to $M$ is the finest non-crossing partition of $[n]$ such that for any $i \in[n]$, the numbers $i$ and $\pi(i)$ are in the same block of $\Pi_{M}$.

Note that if we represent a decorated permutation of $[n]$ by means of its "chord diagram" (see Figure 11, Corollary 3.6 says that the blocks of its corresponding non-crossing partition are the connected components of the diagram.
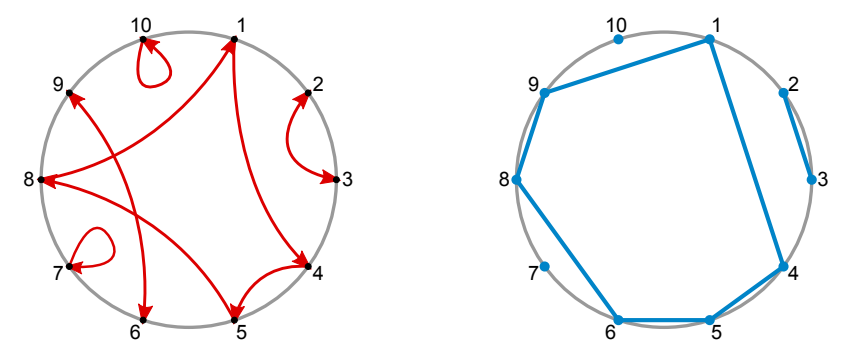

Fig. 1: The chord diagram of a decorated permutation of [10] and its corresponding non-crossing partition.

As a corollary, we obtain a bijection between connected positroids on $[n]$ and an interesting class of permutations of $[n]$.

Definition 3.7 Cal04 A stabilized-interval-free (SIF) permutation $\pi$ of $[n]$ is a permutation which does not stabilize any proper interval of $[n]$; that is, $\pi(I) \neq I$ for all intervals $I \subsetneq[n]$.

Corollary 3.8 For $n \geq 2$, the number of connected positroids on $[n]$ equals the number of SIF permutations on $[n]$.

Having explained the role that non-crossing partitions play in the connectivity of positroids, we use that knowledge to show that the face poset of a positroid polytope lives inside the poset of weighted non-crossing partitions.

Definition 3.9 $A$ weighted non-crossing partition $S^{w}$ of $[n]$ is a non-crossing partition $S$ of $[n]$, say $[n]=$ $S_{1} \sqcup \cdots \sqcup S_{t}$, together with a weight vector $w=\left(w_{1}, \ldots, w_{t}\right) \in\left(\mathbb{Z}_{\geq 0}\right)^{t}$ of integer weights $w_{1}=$ $w\left(S_{1}\right), \ldots, w_{t}=w\left(S_{t}\right)$ with $0 \leq w_{i} \leq\left|S_{i}\right|$ for $i=1, \ldots, t$. The weight of the partition $S^{w}$ is $w_{1}+\cdots+w_{t}$.

The set $N C_{n}$ of non-crossing partitions of $[n]$ is partially ordered by refinement; this poset has many interesting properties and connections to several fields of mathematics. We extend that order to the context of weighted non-crossing partitions. 
Definition 3.10 Let $N C_{n}^{d}$ be the poset of non-crossing partitions of $[n]$ of weight $d$, where the cover relation is given by $S^{w} \lessdot T^{v}$ if

- $T=\left\{T_{1}, \ldots, T_{t}\right\}$ and $S=\left\{T_{1}, \ldots, T_{h-1}, A, T_{h}-A, T_{h+1}, \ldots, T_{t}\right\}$ for some index $1 \leq h \leq t$ and some proper subset $\emptyset \subsetneq A \subsetneq T_{h}$, and

- $v\left(T_{h}\right)=w(A)+w\left(T_{h}-A\right)$ and $v\left(T_{j}\right)=w\left(T_{j}\right)$ for all $j \neq h$.

Let $N C_{n}^{d} \cup\{\widehat{0}\}$ be this poset with an additional minimum element $\widehat{0}$.

The poset $N C_{n}^{d}$ is ranked of height $n$. It has a unique maximal element $\hat{1}$ corresponding to the trivial partition of $[n]$ into one part of weight $d$.

Theorem 3.11 If $M$ is a rank $d$ positroid on [n] then the face poset of the matroid polytope $\Gamma_{M}$ is an induced subposet of $N C_{n}^{d} \cup\{\widehat{0}\}$.

Figure 2 shows the positroid polytope $\Gamma_{M}$ for the positroid $M$ whose bases are $\{12,13,14,23,24\}$. It is a square pyramid. It also shows the face poset of $\Gamma_{M}$, with each face labeled with the corresponding weighted non-crossing partition of [4].

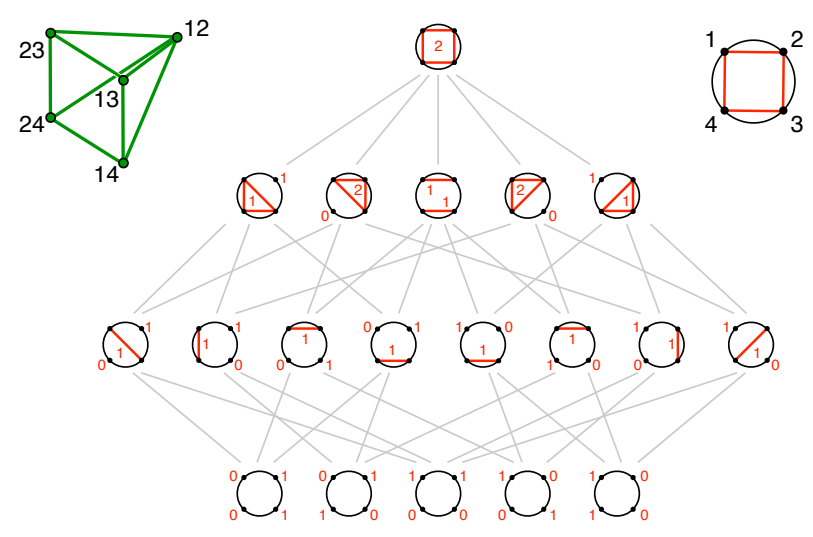

Fig. 2: The face poset of the square pyramid inside $N C_{4}^{2}$.

\section{Enumeration of connected positroids}

In this section we use Theorem 3.2 together with a result of the third author [Wil05], to enumerate connected positroids.

Definition 4.1 Let $p(n)$ be the number of positroids on $[n]$ and $p_{c}(n)$ be the number of connected positroids on $[n]$. Let

$$
P(x)=1+\sum_{n \geq 1} p(n) x^{n} \quad \text { and } \quad P_{c}(x)=1+\sum_{n \geq 1} p_{c}(n) x^{n} .
$$

Many combinatorial objects (such as graphs or matroids) on a set $[n]$ decompose uniquely into connected components $S_{1}, \ldots, S_{k}$, where the partition $[n]=S_{1} \sqcup \cdots \sqcup S_{k}$ has no additional structure. In that case, the Exponential Formula [Sta99, Theorem 5.1.3] tells us that the exponential generating functions 
$E_{t}(x)$ and $E_{c}(x)$ for the total number of objects and the total number of connected objects are related by the formula $E_{c}(x)=\log E_{t}(x)$. In our situation, where the connected components of a positroid form a non-crossing partition, we need the following "non-crossing" analog of the Exponential Formula:

Theorem 4.2 [Bei85. Spe94] Let $K$ be a field. Given a function $f: \mathbb{Z}_{>0} \rightarrow K$ define a new function $h: \mathbb{Z}_{>0} \rightarrow K$ by

$$
h(n)=\sum_{\left\{S_{1}, \ldots, S_{k}\right\} \in N C_{n}} f\left(\# S_{1}\right) f\left(\# S_{2}\right) \cdots f\left(\# S_{k}\right)
$$

where we are summing over all the non-crossing partitions of $[n]$. Define $F(x)=1+\sum_{n \geq 1} f(n) x^{n}$ and $H(x)=1+\sum_{n \geq 1} h(n) x^{n}$. Then

$$
x H(x)=\left(\frac{x}{F(x)}\right)^{\langle-1\rangle},
$$

where $G(x)^{\langle-1\rangle}$ denotes the compositional inverse of $G(x)$.

Corollary 4.3 The generating functions for positroids and connected positroids satisfy:

$$
x P(x)=\left(\frac{x}{P_{c}(x)}\right)^{\langle-1\rangle} .
$$

Enumeration of general positroids has been previously studied by the third author in [Wil05].

Theorem 4.4 We have

$$
P(x)=\sum_{k \geq 0} k ! \frac{x^{k}}{(1-x)^{k+1}}, \quad p(n)=\sum_{k=0}^{n} \frac{n !}{k !}, \quad \lim _{n \rightarrow \infty} \frac{p(n)}{n !}=e .
$$

The following formula also follows easily from the above.

Proposition 4.5 Posb. Prop. 23.2] The exponential generating function for $p(n)$ is

$$
1+\sum_{n \geq 1} p(n) \frac{x^{n}}{n !}=\frac{e^{x}}{1-x} .
$$

The sequence $\{p(n)\}_{n \geq 1}$ is entry A000522 in Sloane's Encyclopedia of Integer Sequences [Slo94]. The first few terms are $2,5,16,65,326,1957,13700, \ldots$.

Theorem 4.6 The number $p_{c}(n)$ of connected positroids on $[n]$ satisfies

$$
\begin{aligned}
p_{c}(n) & =\frac{\left[x^{n}\right] P(x)^{1-n}}{1-n}, \\
p_{c}(n) & =(n-1) p_{c}(n-1)+\sum_{j=2}^{n-2}(j-1) p_{c}(j) p_{c}(n-j) \text { for } n \geq 2, \text { and } \\
\lim _{n \rightarrow \infty} \frac{p_{c}(n)}{n !} & =\frac{1}{e} .
\end{aligned}
$$


The sequence $\left\{p_{c}(n)\right\}_{n \geq 1}$ is, except for the first term, equal to entry A075834 in Sloane's Encyclopedia of Integer Sequences [Slo94]. The first few terms are 2, 1, 2, 7, 34, 206, 1476, ...

Theorem 4.7 If $p(n)$ is the number of positroids on $[n]$ and $p_{c}(n)$ is the number of connected positroids on $[n]$, then

$$
\lim _{n \rightarrow \infty} \frac{p_{c}(n)}{p(n)}=\frac{1}{e^{2}} \approx 0.1353 .
$$

This result is somewhat surprising in view of the conjecture that most matroids are connected:

Conjecture 4.8 (Mayhew, Newman, Welsh, Whittle, [MNWWII]) If $m(n)$ is the number of matroids on $[n]$ and $m_{c}(n)$ is the number of connected matroids on $[n]$, then

$$
\lim _{n \rightarrow \infty} \frac{m_{c}(n)}{m(n)}=1
$$

Theorem 4.7 should not be seen as evidence against Conjecture 4.8 Positroids possess strong structural properties that are quite specific to them. Furthermore, they are a relatively small family of matroids: compare the estimate $\log _{2} \log _{2} m(n) \sim n$ due to Knuth [Knu74] and Bansal, Pendavingh, and van der Pol $[\overline{\mathrm{BPvdP}}]$ with the estimate $p(n) \sim n ! e$, which gives $\log _{2} \log _{2} p(n) \sim \log _{2} n$.

\section{Every positively oriented matroid is realizable}

An oriented matroid is a signed version of the notion of matroid. Just as for matroids, there are several equivalent points of view and axiom systems. For a thorough introduction to the theory of oriented matroids, see $\left[\mathrm{BLVS}^{+} 99\right]$.

Example 5.1 Let $A$ be a $d \times n$ matrix of rank $d$ with entries in an ordered field $K$. For a d-element subset I of $[n]$ we let $\Delta_{I}(A)$ denote the determinant of the $d \times d$ submatrix of $A$ consisting of the columns indexed by $I$. We obtain a chirotope $\chi_{A}:\left(\begin{array}{c}{[n]} \\ d\end{array}\right) \rightarrow\{-1,0,1\}$ by setting

$$
\chi_{A}(I)= \begin{cases}0 & \text { if } \Delta_{I}(A)=0, \\ 1 & \text { if } \Delta_{I}(A)>0, \\ -1 & \text { if } \Delta_{I}(A)<0 .\end{cases}
$$

An oriented matroid $\mathcal{M}=([n], \chi(A))$ arising in this way is called realizable over the field $K$.

Definition 5.2 If $\mathcal{M}=(E, \chi)$ is an oriented matroid, its underlying matroid $\underline{\mathcal{M}}$ is the (unoriented) matroid $\underline{\mathcal{M}}:=(E, \mathcal{B})$ whose bases $\mathcal{B}$ are precisely the sets $\left\{b_{1}, \ldots, b_{d}\right\}$ such that $\chi\left(b_{1}, \ldots, b_{d}\right)$ is nonzero.

Definition 5.3 If $\mathcal{M}=(E, \chi)$ is an oriented matroid, any $A \subseteq E$ induces a reorientation ${ }_{-A} \mathcal{M}:=$ $\left(E,{ }_{-A} \chi\right)$ of $\mathcal{M}$, where ${ }_{-A} \chi$ is the chirotope

$$
-A \chi\left(y_{1}, \ldots, y_{d}\right):=(-1)^{\left|A \cap\left\{y_{1}, \ldots, y_{d}\right\}\right|} \cdot \chi\left(y_{1}, \ldots, y_{d}\right) .
$$

This can be thought of as the oriented matroid obtained from $\mathcal{M}$ by "changing the sign of the vectors in A".

The following definition introduces the class of oriented matroids that is central for our study. 
Definition 5.4 Let $\mathcal{M}=(E, \chi)$ be an oriented matroid of rank $d$ on a set $E$ with a linear order $<$. We say $\mathcal{M}$ is positively oriented with respect to $<$ if there is a reorientation ${ }_{-A} \chi$ that makes all bases positive; that is,

$$
{ }_{-A} \chi(I):={ }_{-A} \chi\left(i_{1}, i_{2}, \ldots, i_{d}\right) \geq 0
$$

for every d-element subset $I=\left\{i_{1}<i_{2}<\ldots<i_{d}\right\} \subseteq E$.

The main result of this section is the following. It answers Conjecture 1.1 in an affirmative way. Its proof is based on Theorem 2.5. which gives a characterization positroids in terms of their matroid polytopes.

Theorem 5.5 Every positively oriented matroid is realizable over $\mathbb{Q}$. Equivalently, the underlying matroid of any positively oriented matroid is a positroid.

\section{The positive matroid Grassmannian is homeomorphic to a ball}

In [Mac93], MacPherson introduced the notion of combinatorial differential manifold, a simplicial pseudomanifold with an additional discrete structure - described in the language of oriented matroids - to model "the tangent bundle." He also developed the bundle theory associated to combinatorial differential manifolds, and showed that the classifying space of matroid bundles is the matroid Grassmannian or MacPhersonian. The matroid Grassmannian therefore plays the same role for matroid bundles as the ordinary Grassmannian plays for vector bundles.

We now introduce the matroid Grassmannian and define its positive analogue. The main result of this section is that the positive matroid Grassmannian is homeomorphic to a closed ball.

There is a natural partial order on oriented matroids called specialization.

Definition 6.1 Suppose that $\mathcal{M}=(E, \chi)$ and $\mathcal{M}^{\prime}=\left(E, \chi^{\prime}\right)$ are two rank $k$ oriented matroids on $E$. We say that $\mathcal{M}^{\prime}$ is a specialization of $\mathcal{M}$, denoted $\mathcal{M} \sim \mathcal{M}^{\prime}$, if (after replacing $\chi$ with $-\chi$ if necessary) we have that

$$
\chi\left(y_{1}, \ldots, y_{k}\right)=\chi^{\prime}\left(y_{1}, \ldots, y_{k}\right) \text { whenever } \chi^{\prime}\left(y_{1}, \ldots, y_{k}\right) \neq 0 .
$$

Definition 6.2 The matroid Grassmannian or MacPhersonian $\operatorname{MacP}(k, n)$ of rank $k$ on $[n]$ is the poset of rank $k$ oriented matroids on the set $[n]$, where $\mathcal{M} \geq \mathcal{M}^{\prime}$ if and only if $\mathcal{M} \leadsto \mathcal{M}^{\prime}$.

One often identifies $\operatorname{MacP}(k, n)$ with its order complex. When we speak of the topology of $\operatorname{Mac} \mathrm{P}(k, n)$, we mean the topology of (the geometric realization of) the order complex of $\operatorname{MacP}(k, n)$, denoted $\|\operatorname{MacP}(k, n)\|$.

MacPherson [Mac93] pointed out that $\|\operatorname{MacP}(k, n)\|$ is homeomorphic to the real Grassmannian $\operatorname{Gr}(k, n)$ if $k$ equals $1,2, n-2$, or $n-1$, but that "otherwise, the topology of the matroid Grassmannian is mostly a mystery." As mentioned in the introduction, Anderson [And99], and Anderson and Davis [AD02] made some progress on this question, obtaining results on the homotopy groups and cohomology of the matroid Grassmannian. However, it is still open whether $\operatorname{MacP}(k, n)$ is homotopy equivalent to $\operatorname{Gr}(k, n)$.

We now introduce a positive counterpart $\mathrm{MacP}^{+}(k, n)$ of the matroid Grassmannian. This space turns out to be more tractable than $\operatorname{MacP}(k, n)$; we can completely describe its homeomorphism type.

Definition 6.3 The positive matroid Grassmannian or positive MacPhersonian $\mathrm{MacP}^{+}(k, n)$ of rank $k$ on $[n]$ is the poset of rank $k$ positively oriented matroids on the set $[n]$, where $\mathcal{M} \geq \mathcal{M}^{\prime}$ if and only if $\mathcal{M} \leadsto \mathcal{M}^{\prime}$. 
For convenience, we usually augment $\mathrm{MacP}^{+}(k, n)$ by adding a least element $\hat{0}$. Our main theorem on the topology of $\mathrm{MacP}^{+}(k, n)$ is the following.

Theorem 6.4 $\mathrm{MacP}^{+}(k, n)$ is the face poset of a regular $C W$ complex homeomorphic to a ball. It follows that:

- $\left\|\mathrm{MacP}^{+}(k, n)\right\|$ is homeomorphic to a ball.

- For each $\mathcal{M} \in \operatorname{MacP}^{+}(k, n)$, the closed and open intervals $\|[\hat{0}, \mathcal{M}]\|$ and $\|(\hat{0}, \mathcal{M})\|$ are homeomorphic to a ball and a sphere, respectively.

- $\mathrm{MacP}^{+}(k, n)$ is Eulerian.

The positive analogue of the real Grassmannian is the positive Grassmannian (also called the totally non-negative Grassmannian). The positive Grassmannian is an example of a positive flag variety, as introduced by Lusztig in his theory of total positivity for real flag manifolds [Lus98], and its combinatorics was beautifully developed by Postnikov [Posb]. The positive Grassmannian has recently received a great deal of attention because of its connection with scattering amplitudes $\left[\mathrm{AHBC}^{+}\right]$.

Definition 6.5 The positive Grassmannian $\operatorname{Gr}^{+}(k, n)$ is the subset of the real Grassmannian where all Plücker coordinates are non-negative.

While it remains unknown whether $\|\operatorname{MacP}(k, n)\|$ is homotopy-equivalent to $\operatorname{Gr}(k, n)$, the positive analogue of that statement is true.

Theorem 6.6 The positive matroid Grassmannian $\left\|\mathrm{MacP}^{+}(k, n)\right\|$ and the positive Grassmannian $\mathrm{Gr}^{+}(k, n)$ are homotopy-equivalent; more specifically, both are contractible, with boundaries homotopyequivalent to a sphere.

Our proofs of Theorems 6.4 and 6.6 are based on the following results on the positive Grassmannian [Posb, Wil07, RW10].

Let $\mathcal{B} \subseteq\left(\begin{array}{c}{[n]} \\ k\end{array}\right)$ be a collection of $k$-element subsets of $[n]$. We define

$$
S_{\mathcal{B}}^{\text {tnn }}=\left\{A \in \mathrm{Gr}^{+}(k, n) \mid \Delta_{I}(A)>0 \text { if and only if } I \in \mathcal{B}\right\} .
$$

Theorem 6.7 Posb] Each subset $S_{\mathcal{B}}^{t n n}$ is either empty or a cell. The positive Grassmannian $\operatorname{Gr}^{+}(k, n)$ is therefore a disjoint union of cells, where $S_{\mathcal{B}^{\prime}}^{t n n} \subset \overline{S_{\mathcal{B}}^{t n n}}$ if and only if $\mathcal{B}^{\prime} \subseteq \mathcal{B}$.

Let $Q(k, n)$ denote the poset of cells of $\operatorname{Gr}^{+}(k, n)$, ordered by containment of closures, and augmented by a least element $\hat{0}$.

Theorem 6.8 Wil07] The poset $Q(k, n)$ is graded, thin, and EL-shellable. It follows that $Q(k, n)$ is the face poset of a regular CW complex homeomorphic to a ball, and that it is Eulerian.

Theorem 6.9 RW10] The positive Grassmannian $\mathrm{Gr}^{+}(k, n)$ is contractible, and its boundary is homotopyequivalent to a sphere. Moreover, the closure of every cell is contractible, and the boundary of every cell is homotopy-equivalent to a sphere.

We have the following result.

Proposition 6.10 For any $k \leq n, \mathrm{MacP}^{+}(k, n)$ and $Q(k, n)$ are isomorphic as posets.

Theorem 6.4 follows from Proposition 6.10 and Theorem 6.8 while Theorem 6.6 follows from Proposition 6.10 and Theorem 6.9 


\section{References}

[AD02] Laura Anderson and James F. Davis, Mod 2 cohomology of combinatorial Grassmannians, Selecta Math. (N.S.) 8 (2002), no. 2, 161-200.

$\left[\mathrm{AHBC}^{+}\right]$Nima Arkani-Hamed, Jacob L. Bourjaily, Freddy Cachazo, Alexander B. Goncharov, Alexander Postnikov, and Jaroslav Trnka, Scattering amplitudes and the positive Grassmannian, preprint, arXiv:1212.5605.

[And99] Laura Anderson, Matroid bundles, New perspectives in algebraic combinatorics (Berkeley, CA, 1996-97), Math. Sci. Res. Inst. Publ., vol. 38, Cambridge Univ. Press, Cambridge, 1999, pp. 1-21.

[Bei85] Janet Simpson Beissinger, The enumeration of irreducible combinatorial objects, Journal of Combinatorial Theory, Series A 38 (1985), no. 2, 143 - 169.

[Bla77] Robert G. Bland, A combinatorial abstraction of linear programming, J. Combinatorial Theory, Ser. B 23 (1977), 33-57.

[BLVS $\left.{ }^{+} 99\right]$ Anders Björner, Michel Las Vergnas, Bernd Sturmfels, Neil White, and Günter M. Ziegler, Oriented matroids, second ed., Encyclopedia of Mathematics and its Applications, vol. 46, Cambridge University Press, Cambridge, 1999.

[BPvdP] N. Bansal, R.A. Pendavingh, and J.G. van der Pol, On the number of matroids, Preprint. arXiv: 1206.6270 .

[BS89] Jurgen Bokowski and Bernd Sturmfels, An infinite family of minor-minimal nonrealizable 3-chirotopes, Mathematische Zeitschrift 200 (1989), 583-589.

[Cal04] David Callan, Counting stabilized-interval-free permutations, J. Integer Seq. 7 (2004), no. 1 , Article 04.1.8, 7 pp. (electronic).

[dS87] Ilda P.F. da Silva, Quelques propriétés des matroides orientés, Ph.D. Dissertation, Université Paris VI, 1987.

[For] Nicolas Ford, The expected codimension of a matroid variety, Preprint. arXiv:1309.0460.

[Knu74] Donald E Knuth, The asymptotic number of geometries, Journal of Combinatorial Theory, Series A 16 (1974), no. 3, 398-400.

[LP] T. Lam and A. Postnikov, Polypositroids, In progress.

[Lus98] G. Lusztig, Total positivity in partial flag manifolds, Represent. Theory 2 (1998), 70-78.

[LV75] Michel Las Vergnas, Matroides orientables, C. R. Acad. Sci. Paris. Ser. A. 280 (1975), 61-64.

[Mac93] Robert MacPherson, Combinatorial differential manifolds, Topological methods in modern mathematics (Stony Brook, NY, 1991), Publish or Perish, Houston, TX, 1993, pp. 203-221. 
[MNW] Dillon Mayhew, Mike Newman, and Geoff Whittle, Is the missing axiom of matroid theory lost forever?, preprint, arXiv:1204.3365.

[MNWW11] Dillon Mayhew, Mike Newman, Dominic Welsh, and Geoff Whittle, On the asymptotic proportion of connected matroids, European J. Combin. 32 (2011), no. 6, 882-890.

[OPS] S. Oh, A. Postnikov, and D. Speyer, Weak separation and plabic graphs, Preprint. arXiv:1109.4434.

[Ox192] James G. Oxley, Matroid theory, Oxford University Press, 1992.

[Posa] Alex Postnikov, Personal communication, 2012.

[Posb] Alexander Postnikov, Total positivity, Grassmannians, and networks, preprint, available at http://www-math.mit.edu/ apost/papers/tpgrass.pdf.

[RW10] Konstanze Rietsch and Lauren Williams, Discrete Morse theory for totally non-negative flag varieties, Adv. Math. 223 (2010), no. 6, 1855-1884.

[Sco06] J. Scott, Grassmannians and cluster algebras, Proceedings of the London Mathematical Society 92 (2006), 345-380.

[Slo94] N. J. A. Sloane, An on-line version of the encyclopedia of integer sequences, Electron. J. Combin 1 (1994), 1-5.

[Spe94] Roland Speicher, Multiplicative functions on the lattice of non-crossing partitions and free convolution, Mathematische Annalen 298 (1994), 611-628 (English).

[Sta99] Richard P. Stanley, Enumerative combinatorics. Vol. 2, Cambridge Studies in Advanced Mathematics, vol. 62, Cambridge University Press, Cambridge, 1999.

[Stu87] Bernd Sturmfels, On the decidability of Diophantine problems in combinatorial geometry, Bull. Amer. Math. Soc. (N.S.) 17 (1987), no. 1, 121-124.

[Vám78] Peter Vámos, The missing axiom of matroid theory is lost forever, J. London Math. Soc. 18 (1978), 403-408.

[Wil05] Lauren K. Williams, Enumeration of totally positive Grassmann cells, Advances in Mathematics 190 (2005), no. 2, $319-342$.

[Wil07] Lauren K. Williams, Shelling totally nonnegative flag varieties, J. Reine Angew. Math. 609 (2007), 1-21. 